\title{
The effect of p-4E-BP1 and p-eIF4E on cell proliferation in a breast cancer model
}

\author{
BERTA PONS $^{1,2^{*}}$, VICENTE PEG $^{1^{*}}$, MARÍA ÁNGELES VÁZQUEZ-SÁNCHEZ ${ }^{1}$, LAURA LÓPEZ-VICENTE ${ }^{1}$, \\ ELISABET ARGELAGUET ${ }^{1}$, LAURA COCH $^{1}$, ALBA MARTÍNEZ ${ }^{1}$, JAVIER HERNÁNDEZ-LOSA ${ }^{1}$, \\ GEMMA ARMENGOL ${ }^{1,3 \#}$ and SANTIAGO RAMON Y CAJAL ${ }^{1 \#}$ \\ ${ }^{1}$ Department of Pathology, Vall d'Hebron University Hospital; ${ }^{2}$ Department of Biochemistry \\ and Molecular Biology, Faculty of Biosciences; ${ }^{3}$ Department of Animal Biology, Plant \\ Biology and Ecology, Universitat Autònoma de Barcelona, Barcelona, Spain
}

Received May 11, 2011; Accepted June 16, 2011

DOI: $10.3892 /$ ijo.2011.1118

\begin{abstract}
Cell signaling pathways and protein translation are crucial for understanding malignant transformation. 4E-BP1 and the eIF4F complex regulate cap-dependent translation. We investigated how 4E-BP1 and eIF4E phosphorylation status affects in vitro and in vivo cell proliferation in a breast cancer model. Cells from 2 breast carcinoma lines (MDA-MB 231 and MDA-MB 468) and human fibroblasts (IMR90 cells) were infected in vitro with a retrovirus carrying a wild-type $4 \mathrm{E}-\mathrm{BP} 1$ or a mutant 4E-BP1 unable to hyperphosphorylate. Overexpression of the mutant 4E-BP1 induced a significant decrease in cell proliferation in IMR90 and MDA-MB 468 cells, but not in MDA-MB 231 cells. A correlation was observed between baseline-phosphorylated eIF4E (p-eIF4E) levels and sensitivity to 4E-BP1 transduction. By co-immunoprecipitation, $\mathrm{p}$-eIF4E seemed to present lower affinity for 4E-BP1 than total eIF4E in MDA-MB 468 cells. After treatment with CGP57380, the MAP kinase-interacting kinase (MNK) inhibitor, downregulation of p-eIF4E levels was associated with an increase of E-cadherin and $\beta$-catenin protein expression. These results provide evidence that $4 \mathrm{E}-\mathrm{BP} 1$ transduction leads to a decrease in cell proliferation, and that high p-eIF4E levels may counteract the suppressor effect of 4E-BP1. We propose that high p-4E-BP1 and p-eIF4E levels are central factors in cell signaling and reflect the oncogenic potential of cell signaling pathways in breast cancer.
\end{abstract}

Correspondence to: Dr S. Ramon y Cajal, Department of Pathology, Vall d'Hebron University Hospital, Pg. Vall d'Hebron 119-129, 08035 Barcelona, Spain

E-mail: sramon@vhebron.net

*Contributed equally

${ }^{\#}$ Contributed equally

Key words: 4E-binding protein 1, eukaryotic initiation factor 4E, breast cancer, protein translation, E-cadherin, $\beta$-catenin

\section{Introduction}

Eukaryotic initiation factor $4 \mathrm{E}$ (eIF4E)-binding protein 1 (4E-BP1) plays a critical role in controlling biological processes, such as protein synthesis, cell survival and growth. Hence, it is not surprising that alterations in 4E-BP1 regulation are associated with tumor development and progression (1). We have previously proposed that the phosphorylated form of this protein (p-4E-BP1) acts as a funnel factor or bottleneck through which the transforming signals converge, channeling the oncogenic proliferative signal (1). Work in our laboratory with a large series of breast, ovarian, prostate and endometrial tumors has shown a correlation between p-4E-BP1 and the clinical and pathologic characteristics of tumors, regardless of the upstream oncogenic alterations (2-4).

Other authors have reported similar results in breast carcinomas, melanomas and prostate cancer (5-8). In a study on breast cancer, phosphorylation of AKT, mTOR and 4E-BP1 was associated with tumor development and progression (7). Transfer of 4E-BP1 phosphorylation site mutants into breast carcinoma cells suppresses their tumorigenicity (9). Kremer et al (8) investigated the expression patterns of several biomarkers of the mTOR pathway in prostate cancer and observed that 4E-BP1 levels, in combination with mTOR and PTEN activation, were among the best biomarkers of prostate intraepithelial neoplasia (PIN). Other reports have associated p-4E-BP1 with patient survival in prostate cancer (5) and melanoma (6).

The importance of 4E-BP1 lies in its control of cap-dependent translation initiation complex assembly. Binding of 4E-BP1 to eIF4E prevents formation of the cap-dependent mRNA translation initiation complex, eIF4F (composed of three subunits, eIF4E, eIF4A and eIF4G), and consequent ribosome binding. $4 \mathrm{E}-\mathrm{BP} 1$ phosphorylation results in eIF4E release, thereby enabling cap-translation initiation. 4E-BP1 is phosphorylated at six serine/threonine sites, and the two ultimate phosphorylations, at Thr70 and Ser65, are considered responsible for eIF4E dissociation. In a specific group of mRNAs, all of them with a lengthy and highly structured 5' UTR, translation is dependent on eIF4E availability. The proteins encoded by these mRNAs, 
such as c-myc, cyclin D1, VEGF and ODC, are related to cell growth and the cell cycle.

The inhibitory binding protein 4E-BP1 regulates eIF4E availability. Accordingly, eIF4E levels are substantially elevated in several types of cancers (10). eIF4E drives malignancy when it is overexpressed in experimental models (11), whereas reducing eIF4E expression effectively inhibits the growth of breast and prostate cancer xenografts, and head and neck squamous carcinoma cells $(12,13)$. Moreover, eIF4E activation is significantly related to reduced patient survival in human prostate cancer, and lung cancer (5). eIF4E can be regulated postranscriptionally by two different mechanisms: binding to translational repressor 4E-BPs and phosphorylation by MNKs (14-16). Although eIF4E phosphorylation on Ser209 was discovered some time ago, it remains unclear whether phosphorylation increases or decreases eIF4E affinity for the cap-structure. Early data indicated that eIF4E phosphorylation increased the affinity for capped mRNAs, whereas other studies have shown that eIF4E phosphorylation actually decreases affinity for the cap-structure and capped oligoribonucleotides (15,17-20).

The aim of this study was to analyze how 4E-BP1 phosphorylation combined with eIF4E phosphorylation affects cell proliferation in vitro and in vivo in a breast cancer model. We show that cells expressing high phosphorylated eIF4E (p-eIF4E) levels can be resistant to blocking of cap-dependent translation with a mutant 4E-BP1 and that downregulation of p-eIF4E associates with an increase of E-cadherin and $\beta$-catenin protein levels.

\section{Materials and methods}

Plasmid constructs. pBABE-4E-BP1wt and pBABE-4E-BP1 (4 Ala) constructs were kindly provided by $\mathrm{Dr} \mathrm{N}$. Sonenberg (McGill University, Montreal, Canada). pBABE-4E-BP1 (4 Ala) contains a mutated form of $4 \mathrm{E}-\mathrm{BP} 1$. The mutations are on the four most relevant 4E-BP1 phosphorylation sites: Thr 37/46, Thr 70 and Ser 65. Both wild-type (wt) and mutant (mut) 4E-BP1 contain two amino terminal hemagglutinin (HA) tags.

Cell lines and cell culture. The following cell lines were used in the study: IMR90 human primary fibroblasts (ATCC, American Type Culture Collection, Middlesex, UK), and two breast carcinoma cell lines, MDA-MB 231 and MDA-MB 468 (ATCC).Cells were maintained in standard DMEM(Dulbecco's modified Eagle's medium) growth medium supplemented with $10 \%$ fetal calf serum and antibiotics. MDA-MB 468 cells were treated with $40 \mu \mathrm{M}$ of MNK inhibitor overnight (CGP 57380, Tocris Bioscience, Ellisville, MO).

Cell transfection and retroviral transduction. To generate cell lines that stably express 4E-BP1 and mutant 4E-BP1, pBABE constructs were transiently transfected into a packaging cell line (GP-293, Clontech, Heidelberg, Germany) according to the manufacturer's instructions. For retroviral infection, cell monolayers were incubated in the presence of the retroviruscontaining supernatant and $4 \mu \mathrm{g} / \mathrm{ml}$ polybrene (Sigma-Aldrich Taufkirchen, Germany) for $24 \mathrm{~h}$. Infection was repeated the next day. Twenty-four hours after the second infection, medium supplemented with puromycin ( $0.7 \mu \mathrm{g} / \mathrm{ml}$, Sigma-Aldrich) was added, and cells underwent selection for 3 days to eliminate uninfected cells.

Growth curves. Two days after selection, cells were counted and seeded in duplicate every 3 days. Population doubling (PD) was determined by the following formula: $\mathrm{PD}=\log \left(\mathrm{N}_{\mathrm{f}} / \mathrm{N}_{\mathrm{i}}\right) / \log 2$, where $\mathrm{N}_{\mathrm{f}}$ is the number of cells counted and $\mathrm{N}_{\mathrm{i}}$ is the number of cells seeded. Cumulative population doubling level (PDL) numbers represent the sum of PDs from previous passages. The number of cells seeded from the IMR90, MDA-MB 468 and MDA-MB 231 lines were $1.4 \times 10^{5}, 1.25 \times 10^{5}$ and $9 \times 10^{4}$ cells, respectively. Each curve was performed at least twice with similar results, and each time point was determined in duplicate.

Western blot analysis. Lysates were obtained from cell lines. Subconfluent cells were lysed in lysis buffer (HEPES $50 \mathrm{mM}$, pH 7.5; NaCl 150 mM; $1 \%$ Triton X-100; EDTA 1 mM; 10\% glycerol) in the presence of protease and phosphatase inhibitors. After clearing the lysates by centrifugation, protein concentrations were determined using the Bradford assay (Bio-Rad Protein Assay, Munich, Germany). About 50-100 $\mu \mathrm{g}$ of protein was denatured and resolved on sodium dodecyl sulfate (SDS) polyacrylamide gel electrophoresis (PAGE) and transferred to polyvinylidene fluoride membranes (PVDF) (Bio-Rad). The primary antibodies used were: anti-4E-BP1 antibody (no. 9452, Cell Signaling, diluted 1:1000), anti-eIF4E (no. 9742, Cell Signaling, diluted 1:1000), anti-p-eIF4E Ser209 (no. 9741, Cell Signaling, diluted 1:500), anti-eIF4G (no. 2498, Cell Signaling, diluted 1:1000), anti-p-eIF4G Ser1108 (no. 2441, Cell Signaling, diluted 1:1000), anti- $\beta$-catenin (no. 9562, Cell Signaling, diluted 1:2000) and anti-HA11 monoclonal antibody (MMS-101P, Covance, Berkeley, CA, diluted 1:1000). Anti-actin (CP01, Calbiochem, Darmstadt, Germany; diluted 1:7000) was used as the loading control. The secondary antibodies used were: donkey anti-rabbit IgG-HRP (NA9340, Amersham Pharma-Biotech, Uppsala, Sweden; diluted 1:2000) and donkey anti-mouse IgG-HRP (NA9340, Amersham Pharma-Biotech; diluted 1:2000). Bound antibodies were visualized with an enhanced chemiluminescence detection kit (Amersham Pharma-Biotech). For the p-eIF4E and p-4E-BP1 band intensities, proteins were quantified by densitometry with the Image J software (version 1.41o, National Institutes of Health, Bethesda, MD), normalized to the intensity of $\beta$-actin in each sample, and expressed in arbitrary densitometric units (ADU).

Immunoprecipitation. Lysed cells were incubated with 4E-BP1 antibody (no. 9452, Cell Signaling, diluted 1:50), eIF4G (no. 2498, Cell Signaling, diluted 1:50), or p-eIF4G Ser1108 (no. 2441, Cell Signaling, diluted 1:50) overnight at $4^{\circ} \mathrm{C}$. The next day, protein G Sepharose 4 Fast Flow (17-0618-01, Amersham Pharma-Biotech) was added, and the solution was gently mixed for $1 \mathrm{~h}$ at $4^{\circ} \mathrm{C}$. Samples were centrifuged at $12,000 \mathrm{x} \mathrm{g}$ for $20 \mathrm{sec}$. The pellet was washed 3 times with $1 \mathrm{ml}$ lysis buffer, and suspended in $30 \mu 1$ sample reducing buffer (1\% SDS, $100 \mathrm{mM}$ DTT, $50 \mathrm{mM}$ Tris, pH 7.5). The suspension was heated to $95^{\circ} \mathrm{C}$ for $3 \mathrm{~min}$ and centrifuged at $12,000 \mathrm{x}$ g for $20 \mathrm{sec}$ to remove the beads. Lastly, $1 \mu \mathrm{l}$ of $0.1 \%$ bromophenol blue was added, and the supernatant was analyzed by Western 
$\mathbf{A}$

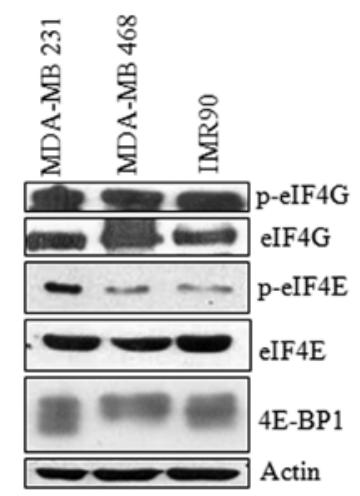

B

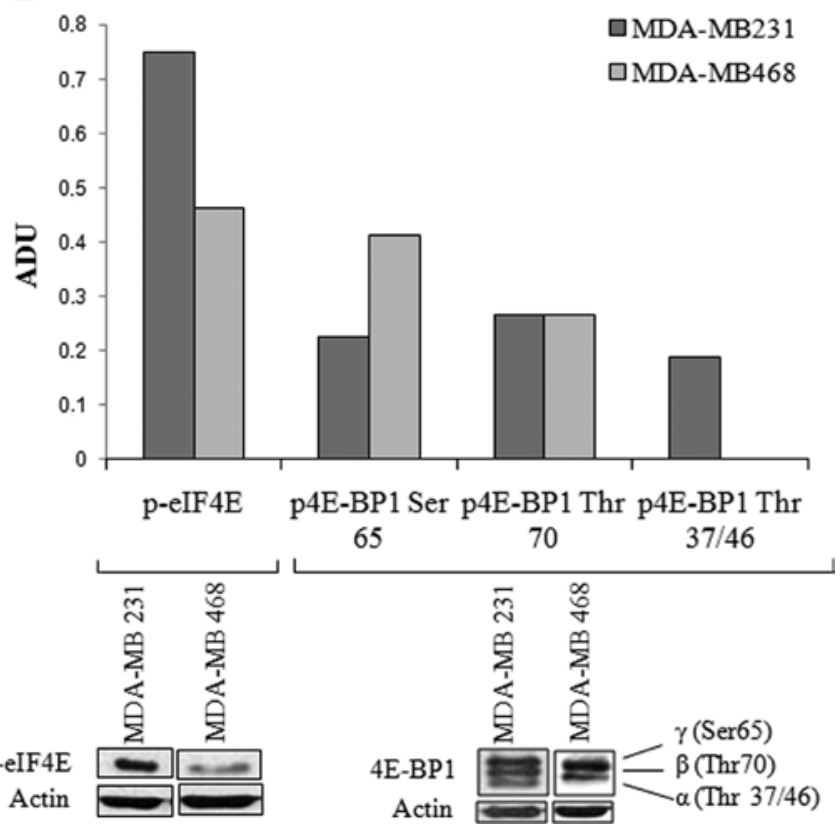

Figure 1.Analysis of the eIF4F translation complex in normal fibroblasts (IMR90) and two breast carcinoma cell lines (MDA-MB 231 and MDA-MB 468). (A) Immunoblot showing p-eIF4G, eIF4G, p-eIF4E, eIF4E and 4E-BP1 protein levels in actively proliferating cells. (B) p-eIF4E and p-4E-BP1 levels calculated by densitometry. MDA-MB 231 cells showed higher p-eIF4E levels and lower p-4E-BP1 Ser65 levels than MDA-MB 468 cells.

blot analysis as described above. These immunoprecipitation experiments were performed three times each, with similar results.

Tumor formation assay. Four-week-old athymic female BALB/C nude mice were purchased from Harlan Laboratories (San Pietro al Natisone, Italy) and housed for 1 week before the experiment. A $5 \times 10^{6}$ amount of MDA-MB 468 or MDA-MB 231 cells was suspended in $100 \mu \mathrm{l}$ of PBS and injected subcutaneously in the flanks of 3 animals (right and left). Tumor growth was monitored by daily measurement. The latency period to tumor formation was defined as the interval from cell inoculation to the time when tumors became visible in at least $50 \%$ of animals. Mice were sacrificed 54 days after injection. The tumor was extracted and weighted.

Statistical analysis. Statistical analyses with the Statistical Package for the Social Sciences (SPSS, version 13.0; SPSS Inc., Chicago, IL) were performed to analyze tumorigenicity. The tumor weights (MDA-MB 468/vector versus MDA-MB 468/4E-BP1 wt and MDA-MB 468/4E-BP1mut, and the same for MDA-MB 231) were compared using the Kruskal-Wallis test. Statistical significance was set at a two-tailed $\mathrm{p} \leq 0.05$.

\section{Results}

Study of the eIF4F complex formation. It is known that the phosphorylation status of 4E-BP1 determines binding to eIF4E. Electrophoresis of 4E-BP1 polyacrylamide gel separates three different isoforms: $\alpha, \beta$ and $\gamma$. The $\gamma$ band represents the most highly phosphorylated band (Ser 65, Thr 70 and Thr 37/46), the $\beta$ band represents phosphorylations at Thr 70 and Thr $37 / 46$, and the $\alpha$ band corresponds to the least phosphorylated form, Thr $37 / 46(9,21,22)$. To assess whether 4E-BP1, eIF4E and eIF4G might be involved in eIF4F complex formation and how their phosphorylation status could affect cell proliferation, we studied two breast carcinoma cell lines (MDA-MB 231 and MDA-MB 468) and human primary fibroblasts (IMR90). Previously, baseline 4E-BP1, eIF4E and eIF4G levels were determined in the three cell lines studied (Fig. 1A). The results showed that $\mathrm{p}$-eIF4E expression levels were lower in MDA-MB 468 than in MDA-MB 231. Conversely, the 4E-BP1 $\gamma$ and $\beta$ band levels were higher in MDA-MB 468. This upward mobility shift reflects hyperphosphorylation of 4E-BP1, which reduces its ability to bind eIF4E and enhances protein translation (Fig. 1B). This could indicate that in MDA-MB 468 cells, which have high p-4E-BP1 levels, most eIF4E should be free to associate with eIF4G and promote eIF4F complex formation.

To assess the relevance of eIF4E phosphorylation when eIF4E binds to 4E-BP1 or eIF4G, we performed immunoprecipitation of MDA-MB 231 and MDA-MB 468 from cell lysates. The results showed that there were some differences between the cell lines. More p-eIF4E was bound to 4E-BP1 in MDA-MB 231 than in MDA-MB 468 (Fig. 2A). However, when eIF4E was analyzed after immunoprecipitation of eIF4G and p-eIF4G, similar results were found in the two cell lines tested. eIF4E and p-eIF4E bound to p-eIF4G at Ser1108 at similar levels in both breast cell lines (Fig. 2B and C).

Overexpression of wild-type and mutant $4 E-B P 1$ in cell lines and its association with cell proliferation and p-eIF4E. The phosphorylation status of 4E-BP1 determines the affinity for eIF4E. Presence of phosphates on the Thr 37/46 residues is necessary to phosphorylate the protein's carboxy-terminal sites, Thr 70 and Ser 65. To determine the importance of these phosphorylation sites on cell proliferation, the two breast cancer lines and IMR90 were infected with retroviral vectors carrying a pBABE-4E-BP1wt and pBABE-4E-BP1 (4 Ala). 4E-BP1 (4 Ala) is a mutated variant of the protein in which Thr37/46, Thr70 and Ser65 are replaced by Ala. The resulting mutated 4E-BP1, 4E-BP1mut, binds constitutively to eIF4E and inhibits translation initiation. 


\section{A. IP: 4E-BP1}

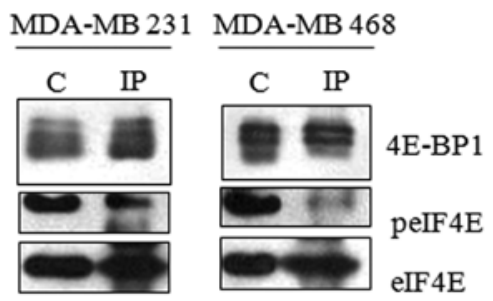

\section{B. IP: eIF4G}

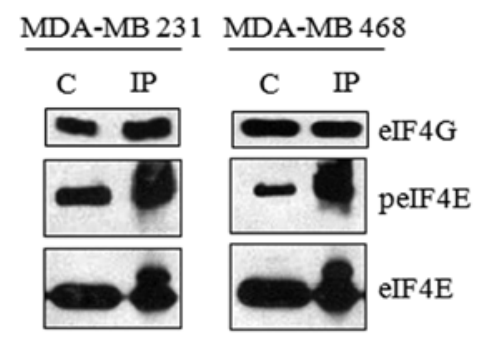

C. IP: p-eIF4G

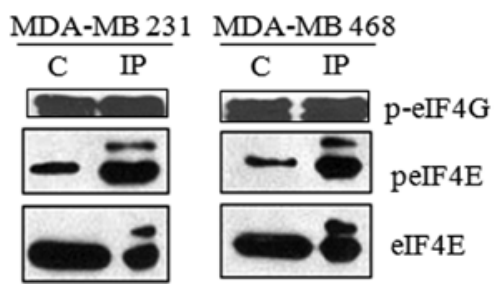

Figure 2. Co-immunoprecipitation of 4E-BP1, eIF4G and p-eIF4G with eIF4E and p-eIF4E in malignant breast epithelial cells. (A) eIF4E and p-eIF4E were detected by Western blot analysis in immunoprecipitation with 4E-BP1. The unprecipitated sample is the control. The immunoprecipitated protein target 4E-BP1 is shown by Western blot analysis. (B) Immunoprecipitation with eIF4G. eIF4E and p-eIF4E binding was detected by Western blot analysis. The immunoprecipitated protein target eIF4G is shown by Western blot analysis. (C) Immunoprecipitation with p-eIF4G. eIF4E and p-eIF4E binding was detected by Western blot analysis. The immunoprecipitated protein target p-eIF4G is shown by Western blot analysis. The same blot was probed first for p-eIF4E and then for total eIF4E. C, control; IP, immunoprecipitation.

In IMR90 cells, 4E-BP1 expression led to a decrease in proliferation compared to cells expressing the empty vector. Moreover, the effect on cell proliferation was higher when the constitutive translational repressor 4E-BP1mut was expressed (Fig. 3A). In breast carcinoma cells, the proliferation decrease after 4E-BP1 overexpression was higher in MDA-MB 468 than in MDA-MB 231 cells, which did not show any significant effect after 4E-BP1 transduction (Fig. 3B and C). The differences in molecular stoichiometry observed in these cell lines, such as hyperphosphorylation of 4E-BP1 and low p-eIF4E levels in MDA-MB 468 and IMR90 cells, could explain the differences observed after 4E-BP1 transduction. MDA-MB 231 cells, which were resistant to the translational repressor, presented low p-4E-BP1 levels and high p-eIF4E levels. This difference may be explained by variations in the signaling pathways of these cells due to their activation status or molecular stoichiometry. MDA-MB 468 cells harbor mutations in PTEN and high levels of p-4E-BP1, and MDA-MB 231 cells have KRAS mutations and high p-eIF4E.

In addition, the ratio of hypophosphorylated $4 \mathrm{E}-\mathrm{BP} 1$ to eIF4E was calculated by densitometry. Cells with decreased proliferation, such as MDA-MB 468 cells with 4E-BP1 wt or mut, had a ratio close to 1 , indicating a lack of free eIF4E for translation initiation, whereas proliferative cells presented ratios below 0.6, indicating abundant free eIF4E (Fig. 3D). Hemagglutinin immunoblotting confirmed ectopic HA 4E-BP1 expression, whereas cells infected with null vector showed no HA expression.

Effect of p-eIF4E on cell proliferation. Because MDA-MB 231 and MDA-MB 468 cells presented different baseline p-eIF4E levels, we analyzed the status of eIF4E phosphorylation in cells that overexpressed the pBABE-4E-BP1 wt and mutant 4E-BP1 form [pBABE-4E-BP1 (4 Ala)]. The two cell lines tested showed some differences. MDA-MB 468 cells transduced with pBABE-4E-BP1 (4 Ala) and pBABE-4E-BP1 wt showed downregulation of eIF4E phosphorylation compared to cells transduced with pBABE null, the effect being more evident after pBABE-4E-BP1 (4 Ala) transduction, where p-eIF4E levels were almost undetectable after 7 days. Conversely, MDA-MB 231 cells did not show any differences in p-eIF4E levels after 4E-BP1 transduction (Fig. 4A).

As is described in Fig. 3B, overexpression of wt and mutant 4E-BP1 in MDA-MB 468 caused a decrease in cell proliferation and correlated with the low levels of p-eIF4E. We then set out to determine whether p-eIF4E level was associated with in vitro cell growth. MDA-MB 468 and MDA-MB 231 cells were treated with the MNK inhibitor CGP57380 to inhibit eIF4E phosphorylation. p-eIF4E was downregulated after $12 \mathrm{~h}$ (Fig. 4B). Interestingly, cells showed decreased growth and formed much more compact, three-dimensional plaques after inhibitor treatment. The differences relative to untreated cells were evident: cells with p-eIF4E showed more irregular cell clusters and spreading out of cells from the clusters. These cytological features are similar to those observed in well differentiated adenocarcinomas, and this suggested that some cell adhesion proteins, such as E-cadherin and/or $\beta$-catenin might be upregulated. To check this hypothesis, Western blotting was used to analyze E-cadherin and $\beta$-catenin levels in MDA-MB 468 cells and $\beta$-catenin in MDA-MB 231 cells (these cells do not express E-cadherin) (Fig. 4B). The results showed an increase in the two proteins after CGP57380 treatment. Similar results were observed in a colon carcinoma cell line (HT-29) (data not shown). To correlate E-cadherin and $\beta$-catenin expression with p-eIF4E levels, we analyzed levels of these factors in MDA-MB 468 cells overexpressing pBABE-4E-BP1 (4 Ala) after 7 days post-transduction, when p-eIF4E was almost undetectable. Interestingly, at this time, MDA-MB 468 cells infected with pBABE-4E-BP1 (4Ala) displayed increased E-cadherin and $\beta$-catenin expression, similar to the effect observed after inhibition of p-eIF4E with CGP57380 (Fig. 4C).

Xenograft tumor growth. To investigate the effects of 4E-BP1 in vivo, MDA-MB 231 and MDA-MB 468 cells transduced with pBABE-4E-BP1 wt or pBABE-4E-BP1 (4 Ala) were injected into the flanks of nude mice, and tumor growth was evaluated. 


\section{A IMR90}

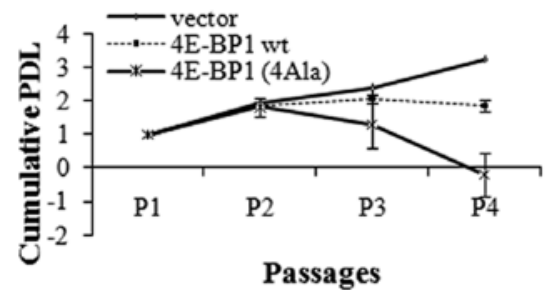

\section{B $\quad$ MDA-MB 468}

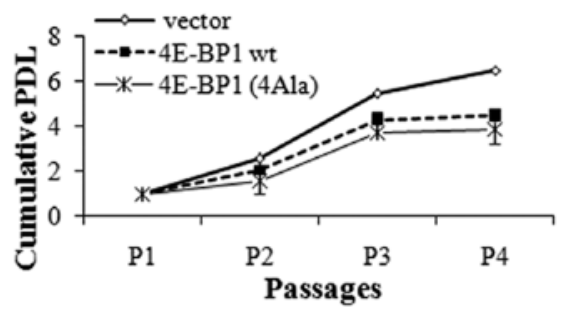

\section{MDA-MB 231}
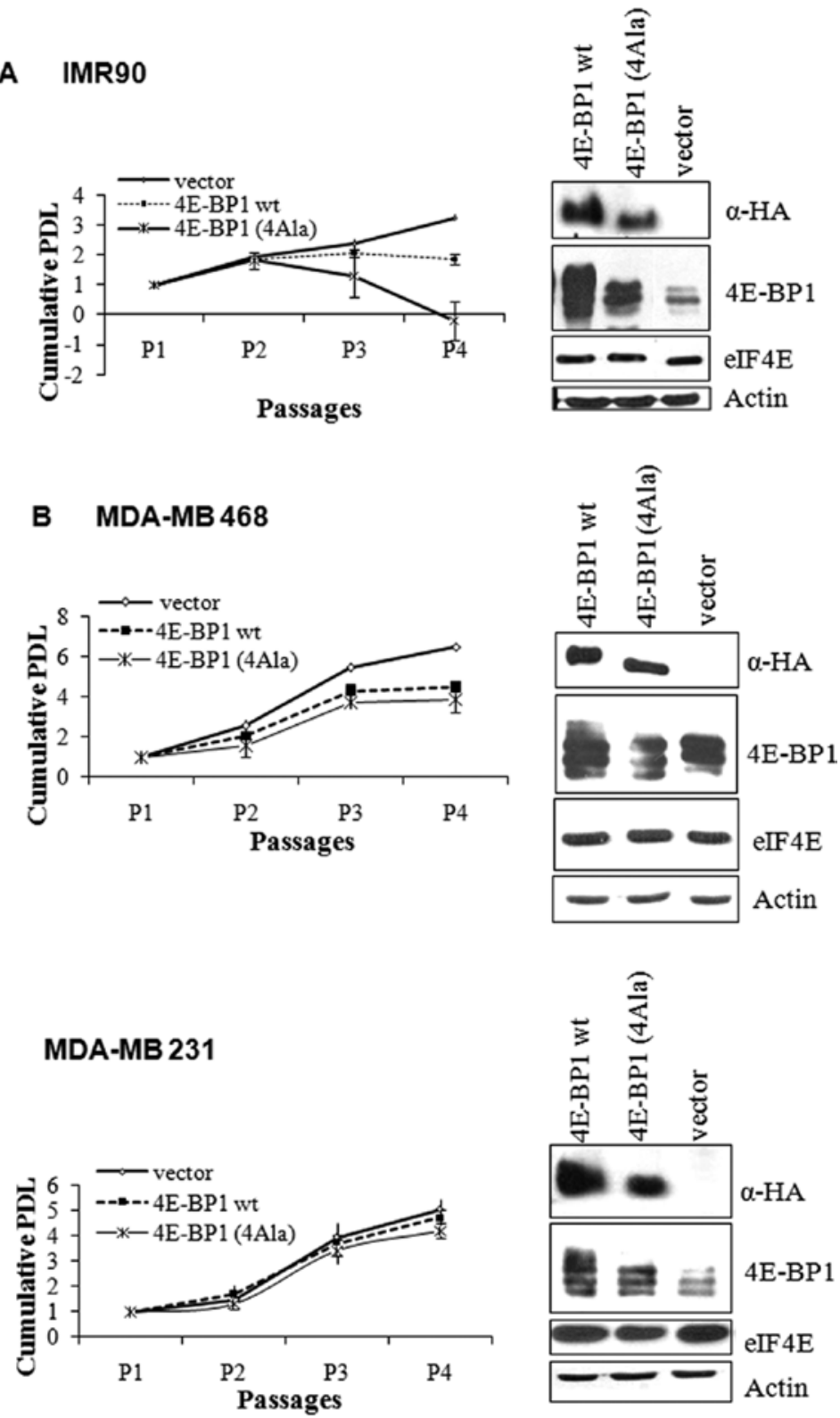

D

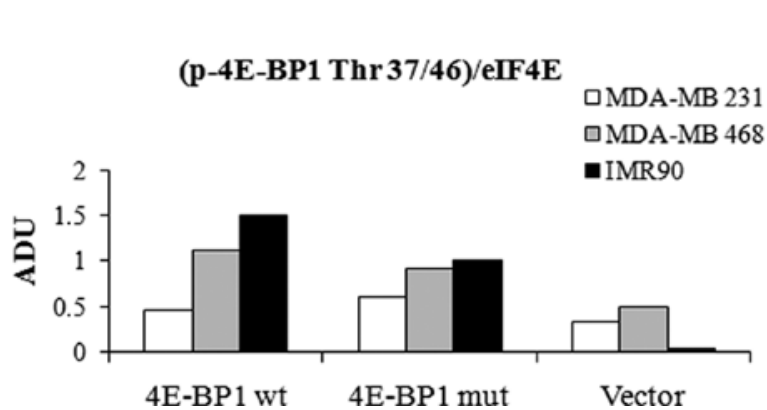

Figure 3. Effect of wild-type and mutant 4E-BP1 (4E-BP1wt and 4E-BP1 mut, respectively) on cell proliferation of stably transduced cells. (A) Proliferation of IMR90 cells was significantly suppressed when 4E-BP1wt and 4E-BP1mut were overexpressed. (B) Proliferation of MDA-MB 468 cells was suppressed when 4E-BP1wt and 4E-BP1mut were overexpressed. (C) There was no effect on MDA-MB 231 proliferation when cells were transduced with 4E-BP1wt or 4E-BP1mut. In all cases, ectopic expression of 4E-BP1 was confirmed by hemagglutinin immunoblotting. (D) Hypophosphorylated 4E-BP1/eIF4E ratio calculated by densitometry. Cells with decreased proliferation, such as MDA-MB 468 cells with 4E-BP1wt or 4E-BP1 (4Ala), had a ratio close to 1.

MDA-MB 231 cells expressing the null vector and 4E-BP1wt formed tumors with a latency period of 5 days, whereas cells expressing 4E-BP1mut formed tumors with a longer latency period (12 days). However, differences in tumor size along the 
A

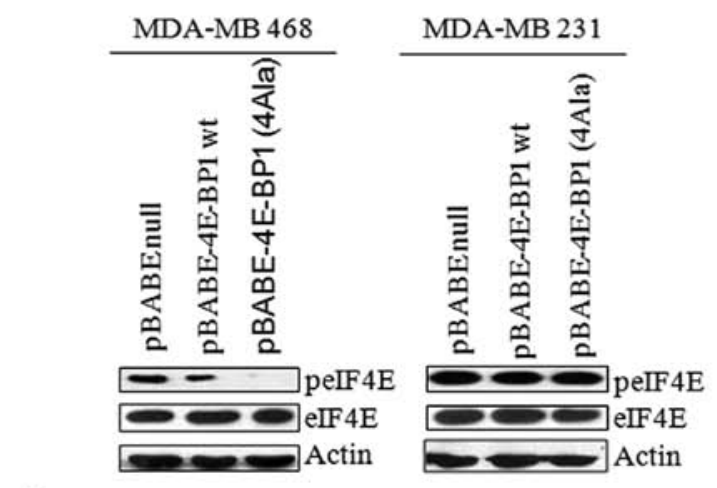

B
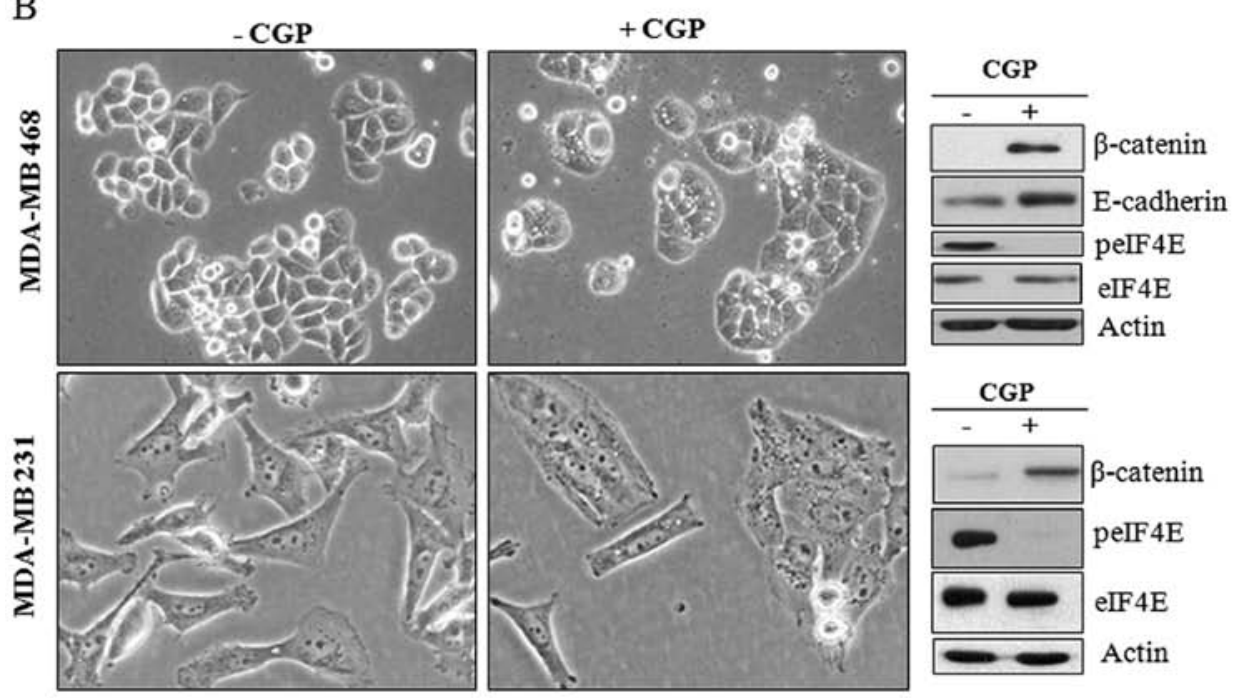

$\mathrm{C}$

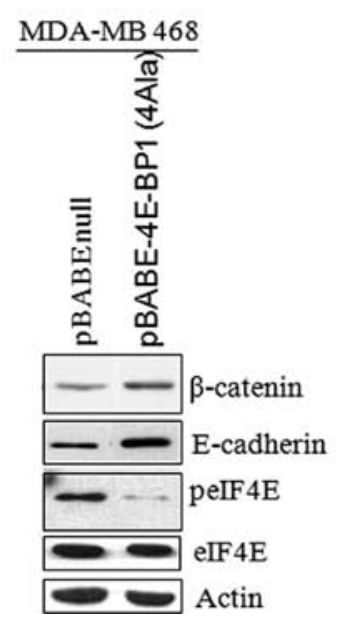

Figure 4. Effect of the p-eIF4E on cell proliferation. (A) Status of eIF4E phosphorylation on MDA-MB 468 cells and MDA-MB 231 cells after overexpression of wt and mutant 4E-BP1. (B) MDA-MB 468 and MDA-MB 231 cells after treatment with MNK inhibitor (CGP 57380). eIF4E phosphorylation was blocked after treatment. An increase of E-cadherin and $\beta$-catenin was observed after treatment. (C) Analysis of E-cadherin and $\beta$-catenin by Western blot analysis. MDA-MB 468 pBABE-4E-BP1 (4Ala) cells 7 days after transduction showed an increase of E-cadherin and $\beta$-catenin.

study time course were not statistically significant (Fig. 5A). MDA-MB 468 cells formed tumors with a latency period of 7 days, whereas cells expressing 4E-BP1wt or 4E-BP1mut formed tumors with much longer latency. MDA-MB 468 tumors formed by $4 \mathrm{E}-\mathrm{BP} 1 \mathrm{wt}$ cells were first apparent on day 12 , whereas tumors formed by $4 \mathrm{E}-\mathrm{BP} 1 \mathrm{mut}$ did not appear until day 26 after injection. In the MDA-MB 468 cell model, tumors expressing 4E-BP1wt or 4E-BP1mut were significantly smaller than tumors with empty vector at days 7 and 26 after injection (Kruskal-Wallis test, $\mathrm{p}=0.0042, \mathrm{p}=0.0035$, respectively) (Fig. 5B). Moreover, tumor size in mice with 4E-BP1mut was statistically different from that of mice with the empty vector at day 54 (Kruskal-Wallis test, $\mathrm{p}=0.0128$ ), even though small tumors were already observed. Conversely, the tumor 
A
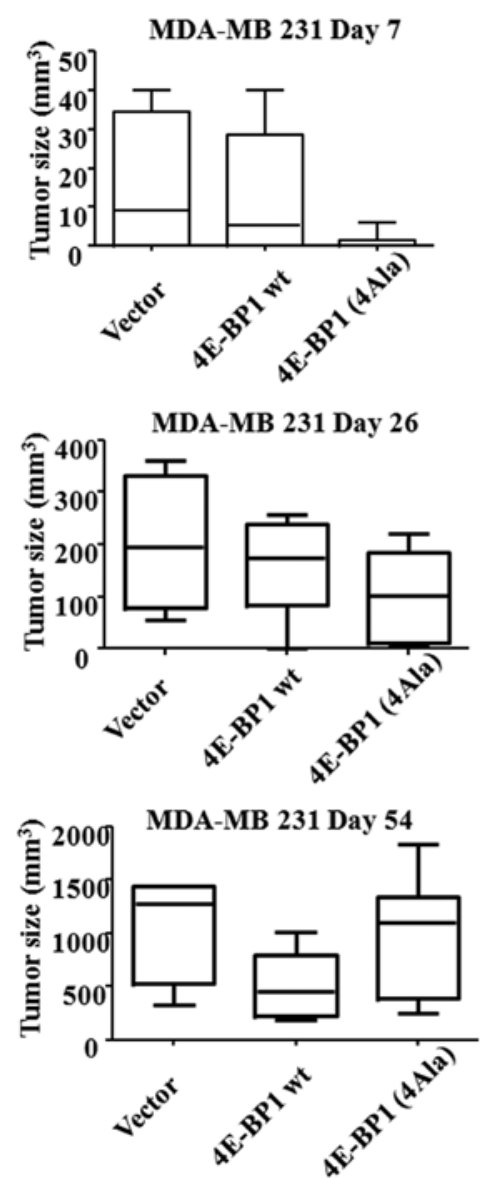

B
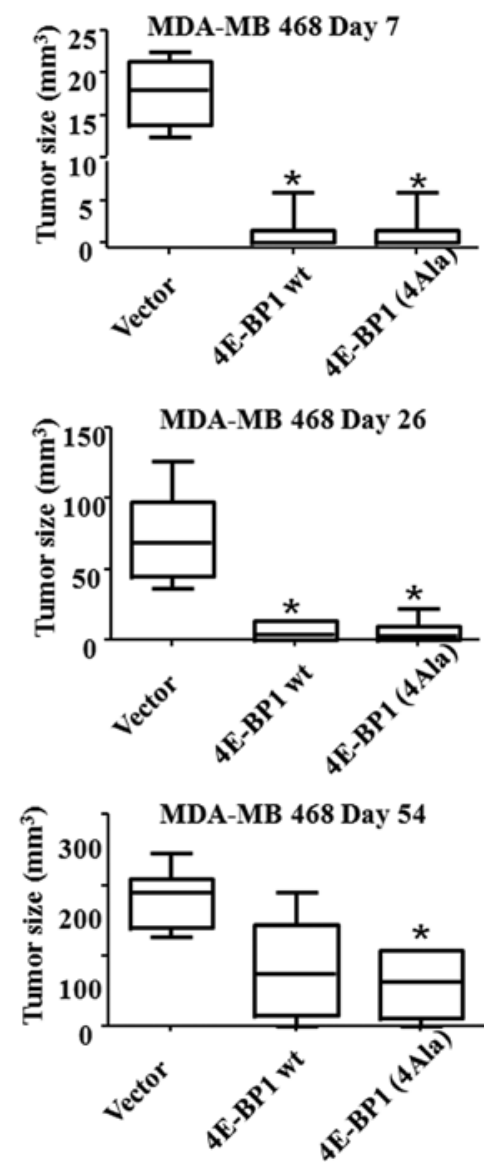

Figure 5. Xenograft tumor growth. MDA-MB 231 cells $\left(5 \times 10^{6}\right)$ transduced with wild-type 4E-BP1 (4E-BP wt) or 4E-BP1 with mutations at the Thr37, Thr46, Thr70 and Ser65 phosphorylation sites [4E-BP1 (4 Ala)] were injected into the back of nude mice, and tumor growth was evaluated at the time intervals indicated. (A) MDA-MB 231 tumor volume on days 7, 26 and 54 after cancer cell injection. Wild-type 4E-BP1 and mutated 4E-BP1 show no significant effect. (B) MDA-MB 468 tumor volume on day $7(\mathrm{p}=0.0042)$ and $26(\mathrm{p}=0.0035)$ after cancer cell injection. At day 54 , the differences in tumor volume were only significant in cells expressing 4E-BP1 (4 Ala) compared with mice with the empty vector ( $\mathrm{p}=0.0128$ ). The differences in mice expressing 4E-BP1 wt and mice with empty vector were not statistically significant.

size in mice with 4E-BP1 wt at day 54 was not statistically significantly smaller compared to mice with the empty vector (pBABE). This could suggest that the power of tumor formation is dependent on loss of the hypophosphorylated forms of 4E-BP1; the differences in tumor size in mice with 4E-BP1 mutant, which cannot be phosphorylated, were evident until the end of the experiment.

\section{Discussion}

The findings of this study further support the concept that high levels of p-4E-BP1 and eIF4E are associated with cell proliferation $(1-4,7,16,23,24)$. Moreover, our data provide new insight into the role of p-eIF4E in human carcinoma cell lines and the association of this factor with tumor aggressiveness and resistance to the tumor-suppressor effect of 4E-BP1.

We performed in vivo and in vitro experimental analyses with a wt and a mutated form of 4E-BP1 at phosphorylation sites that bind to eIF4E, blocking eIF4E activity. In normal cells (IMR90 fibroblasts) ectopic expression of 4E-BP1wt suppressed cell proliferation. In addition, pBABE-4E-BP1 (4Ala) transduction induced a decrease in cell proliferation relative to that of cells with 4E-BP1wt. It is important to note that binding of eIF4E with mutant 4E-BP1, which cannot be phosphorylated at sites Thr37, Thr46, Thr70, or Ser65, induced a clear loss of viability in normal cells, where cap-dependent translation seems to be crucial (9). In malignant cells, cell death did not occur after transduction of 4E-BP1mut, but cell proliferation decreased and tumorigenicity was delayed. This effect was stronger in MDA-MB 468 breast cancer cells than in MDA-MB 231, where no significant effect was observed. Interestingly, the in vitro and in vivo experiments showed similar results. A previous study by Avdulov et al (9) also reported in vitro antiproliferative activity of 4E-BP1 in the same breast carcinoma cells used in the present study. Furthermore, these authors observed that transfer of 4E-BP1 phosphorylation site mutants into MDA-MB 468 cells suppressed their tumorigenicity. However, the mutant used in that study was A37/A46, whereas in the present study a mutant in all the 4E-BP1 phosphorylation sites was used, since the two ultimate phosphorylations, at Thr70 and Ser65, are considered responsible for eIF4E dissociation.

Our in vivo experiments were stopped at 54 days post-injection. We found that tumorigenicity suppression is maintained 
up to 54 days in MDA-MB 468 cells with 4E-BP1mut. In MDA-MB 231 cells, suppression of tumorigenicity was not so evident and disappeared by day 54. Interestingly, MDA-MB 468 cells harbor mutations at the PTEN gene, which most probably is constitutively activating mTOR and therefore, phosphorylation of 4E-BP1, as was observed. We suggest that these cells are more sensitive to 4E-BP1 phosphorylation than MDA-MB 231 cells, which had lower baseline levels of Ser65 4E-BP1. It is likely that hypophosphorylated 4E-BP1 bound to eIF4E; hence, overexpression of 4E-BP1 (normal or mutated) had no effect on cell growth. We observed that cells with decreased proliferation, such as MDA-MB 468 cells with 4E-BP1 wt or mut, had a ratio close to 1 , which indicates a lack of free eIF4E for translation initiation. On the other hand, proliferative cells presented ratios below 0.6 , indicating abundant free eIF4E.

Moreover, MDA-MB 231 cells could be using cell signaling pathways other than 4E-BP1 phosphorylation. Of note, these cells have mutations at the KRAS gene and therefore, could be more MAPK-dependent.

Because we also observed some differences in baseline p-eIF4E in the two breast carcinoma lines studied, we extended our studies in an attempt to understand the contribution of p-eIF4E in enhancing cell proliferation. The biochemical pathways underlying the role of p-eIF4E in malignant tumors remain unclear and the biological significance of eIF4E phosphorylation and its effect on translation is controversial $(15,18,25)$. eIF4E phosphorylation is dispensable for mouse development (26), but seems to be important for normal development in Drosophila (27). In a cell culture study by Borden et al, nuclear eIF4E phosphorylation was seen to enhance the mRNA transport function and transformation activity of eIF4E (28), and eIF4E is reported to promote export of ODC and cyclin D1 from the nucleus to the cytoplasm (29). Thus, phosphorylation of nuclear eIF4E seems to be an important step in mRNA transport control, and consequently, in the transforming properties of eIF4E. Tumorigenesis in transgenic eIF4E-expressing mice is markedly increased, leading to the development of lymphomas (30). This transforming activity of eIF4E has been associated with its phosphorylation $(18,31)$ and with c-myc co-activation (30). In fact, eIF4E phosphorylation has been linked to suppression of apoptosis by an increase in the antiapoptotic protein MCL1 (18).

Another biochemical mechanism that could explain the role of p-eIF4E in transformation is the fact that p-eIF4E does not bind as strongly to 4E-BP1 as total eIF4E does, at least in some cell types. Previously it was reported that p-eIF4E binds more efficiently to capped mRNAs (17), suggesting that the affinity of phosphorylated eIF4E for 4E-BP1 is decreased. Moreover, Chen et al (19) reported that p-eIF4E binds less well to 4E-BP1 than the unphosphorylated form. In our coimmunoprecipitation and Western blot studies, p-eIF4E seemed to show a weaker affinity for 4E-BP1 than total eIF4E in MDA-MB 468 cells, but not in MDA-MB 231 cells. MDA-MB 468 were much more sensitive to the suppressor effect of 4E-BP1mut, and this sensitivity correlated with baseline p-eIF4E levels. MDA-MB 231 cells presented higher p-eIF4E levels than MDA-MB 468 cells. Moreover, when 4E-BP1wt and mutant were overexpressed in MDA-MB 468 cells, p-eIF4E levels clearly decreased. In a more extensive study, we also observed that high levels of peIF4E associate with resistance to over- expression of 4E-BP1 in some carcinoma cell lines (data not shown) and support the idea that lower affinity of p-eIF4E to 4E-BP1 could be related to a biochemical pathway to override the suppressor effect of 4E-BP1.

eIF4E is phosphorylated by the MNKs, which are activated through the classic MAP kinase pathway (ERK1, 2) and the stress-activated MAP kinase pathway (p38 MAP kinase) $(15,26,32)$. In addition to eIF4E, other MNK substrates (e.g. hnRNPA1) are involved in translational control (33), and MNKs seem to play a crucial role in regulation of specific mRNAs, rather than in general translation (34). Because treatment with MNK inhibitor induced a decrease in cell growth in our model and cells acquired more compact clusters, we hypothesized that treated cells might overexpress cell adhesion proteins, such as E-cadherin and $\beta$-catenin (35). Effectively, we found that the entire cell membrane of MDA-MB 468 strongly expressed E-cadherin and $\beta$-catenin after treatment with the inhibitor. A similar pattern was observed in MDA-MB 468 cells transduced with pBABE-4E-BP1 (4Ala), 7 days after transduction, which exhibited lower levels of p-eIF4E. Interestingly, in MDA-MB 231 cells, which lack expression of E-cadherin, an increase of $\beta$-catenin was detected after CGP57380 treatment. These findings could explain the more aggressive behavior of tumors harboring high levels of p-eIF4E and its association with a more mesenchymal phenotype. However, further studies are needed to describe the biochemical pathways and factors involved in upregulation of E-cadherin and $\beta$-catenin expression levels observed after treatment with an MNK inhibitor.

In summary, our results show that IMR90 and MDA-MB 468 breast cancer cell lines were sensitive to 4E-BP1 transduction and it correlates with low p-eIF4E levels. We propose that high levels of p-4E-BP1, which does not bind to eIF4E, and high levels of p-eIF4E, which can likely bypass the suppressor effect of $4 \mathrm{E}-\mathrm{BP} 1$, are central factors in cell signaling and reflect the oncogenic potential of these pathways in breast cancer. These results provide evidence that 4E-BP1 and eIF4E phosphorylation inhibitors could be a new group of molecular funnel targets for breast cancer treatment.

\section{Acknowledgments}

We are grateful to the tumor banks of Hospital Vall d'Hebron and Xarxa del Banc de Tumors de Catalunya for providing the tumor samples. Our thanks to Teresa Moliné for technical assistance, J.L. Parra and N. Sonenberg for their careful reading and suggestions, and $\mathrm{C}$. Cavallo for English editing. Grant support: Fondo de Investigaciones Sanitarias (ref. 05/0818 and 08/0143), Fundació Marató TV3 (ref. 052710), Mutua Madrileña (FMMA/2009/02), Redes temáticas de Investigación Cooperativa en Salud (ref. RD06/0020/0104 and RD06/0020/1020) and Generalitat de Catalunya (ref. 2005SGR00144).

\section{References}

1. Armengol G, Rojo F, Castellvi J, et al: 4E-binding protein 1: a key molecular funnel factor in human cancer with clinical implications. Cancer Res 67: 7551-7555, 2007.

2. Rojo F, Najera L, Lirola J, et al: 4E-binding protein 1, a cell signaling hallmark in breast cancer that correlates with pathologic grade and prognosis. Clin Cancer Res 13: 81-89, 2007. 
3. Castellvi J, Garcia A, Rojo F, et al: Phosphorylated 4E binding protein 1: a hallmark of cell signaling that correlates with survival in ovarian cancer. Cancer 107: 1801-1811, 2006.

4. Castellvi J, Garcia A, Ruiz-Marcellan C, et al: Cell signaling in endometrial carcinoma: phosphorylated 4E-binding protein-1 expression in endometrial cancer correlates with aggressive tumors and prognosis. Hum Pathol 40: 1418-1426, 2009.

5. Graff JR, Konicek BW, Lynch RL, et al: eIF4E activation is commonly elevated in advanced human prostate cancers and significantly related to reduced patient survival. Cancer Res 69: 3866-3873, 2009.

6. O'Reilly KE, Warycha M, Davies MA, et al: Phosphorylated 4E-BP1 is associated with poor survival in melanoma. Clin Cancer Res 15: 2872-2878, 2009.

7. Zhou X, Tan M, Stone Hawthorne V, et al: Activation of the Akt/mammalian target of rapamycin/4E-BP1 pathway by ErbB2 overexpression predicts tumor progression in breast cancers. Clin Cancer Res 10: 6779-6788, 2004.

8. Kremer CL, Klein RR, Mendelson J, et al: Expression of mTOR signaling pathway markers in prostate cancer progression. Prostate 66: 1203-1212, 2006.

9. Avdulov S, Li S, Michalek V, et al: Activation of translation complex eIF4F is essential for the genesis and maintenance of the malignant phenotype in human mammary epithelial cells. Cancer Cell 5: 553-563, 2004.

10. Mamane Y,Petroulakis E,LeBacquer O and Sonenberg N: mTOR, translation initiation and cancer. Oncogene 25: 6416-6422, 2006.

11. Schneider RJ and Sonenberg N: Translational control in cancer development and progression. In: Translational Control in Biology and Medicine. Mathews M, Sonenberg N and Hershey JWB (eds) Cold Spring Harbor Laboratory Press, New York, pp401-432, 2007.

12. Oridate N, Kim HJ, Xu X and Lotan R: Growth inhibition of head and neck squamous carcinoma cells by small interfering RNAs targeting eIF4E or cyclin D1 alone or combined with cisplatin. Cancer Biol Ther 4: 318-323, 2005.

13. Graff JR, Konicek BW, Vincent TM, et al: Therapeutic suppression of translation initiation factor eIF4E expression reduces tumor growth without toxicity. J Clin Invest 117: 2638-2648, 2007.

14. Richter JD and Sonenberg N: Regulation of cap-dependent translation by eIF4E inhibitory proteins. Nature 433: 477-480, 2005.

15. Scheper GC and Proud CG: Does phosphorylation of the capbinding protein eIF4E play a role in translation initiation? Eur J Biochem 269: 5350-5359, 2002.

16. Coleman LJ, Peter MB, Teall TJ, et al: Combined analysis of eIF4E and 4E-binding protein expression predicts breast cancer survival and estimates eIF4E activity. Br J Cancer 100: 1393-1399, 2009.

17. Minich WB, Balasta ML, Goss DJ and Rhoads RE: Chromato graphic resolution of in vivo phosphorylated and nonphosphorylated eukaryotic translation initiation factor eIF-4E: increased cap affinity of the phosphorylated form. Proc Natl Acad Sci USA 91: 7668-7672, 1994.

18. Wendel HG, Silva RL, Malina A, et al: Dissecting eIF4E action in tumorigenesis. Genes Dev 21: 3232-3237, 2007.

19. Chen YJ, Tan BC, Cheng YY, Chen JS and Lee SC: Differential regulation of CHOP translation by phosphorylated eIF4E under stress conditions. Nucleic Acids Res 38: 764-777, 2009.
20. Zuberek J, Wyslouch-Cieszynska A, Niedzwiecka A, et al: Phosphorylation of eIF4E attenuates its interaction with mRNA 5 ' cap analogs by electrostatic repulsion: intein-mediated protein ligation strategy to obtain phosphorylated protein. RNA 9: 52-61, 2003.

21. Constantinou C and Clemens MJ: Regulation of the phosphorylation and integrity of protein synthesis initiation factor eIF4GI and the translational repressor $4 \mathrm{E}-\mathrm{BP} 1$ by $\mathrm{p} 53$. Oncogene 24 : 4839-4850, 2005.

22. Yang DQ and Kastan MB: Participation of ATM in insulin signalling through phosphorylation of eIF-4E-binding protein 1. Nat Cell Biol 2: 893-898, 2000.

23. De Benedetti A and Harris AL: eIF4E expression in tumors: it possible role in progression of malignancies. Int J Biochem Cell Biol 31: 59-72, 1999

24. De Benedetti A and Graff JR: eIF-4E expression and its role in malignancies and metastases. Oncogene 23: 3189-3199, 2004.

25. Ma XM and Blenis J: Molecular mechanisms of mTOR-mediated translational control. Nat Rev Mol Cell Biol 10: 307-318, 2009.

26. Ueda $T$, Watanabe-Fukunaga $R$, Fukuyama $H$, Nagata $S$ and Fukunaga R: Mnk2 and Mnk1 are essential for constitutive and inducible phosphorylation of eukaryotic initiation factor $4 \mathrm{E}$ but not for cell growth or development. Mol Cell Biol 24: 6539-6549, 2004.

27. Lachance PE, Miron M, Raught B, Sonenberg N and Lasko P: Phosphorylation of eukaryotic translation initiation factor $4 \mathrm{E}$ is critical for growth. Mol Cell Biol 22: 1656-1663, 2002.

28. Topisirovic I, Ruiz-Gutierrez M and Borden KL: Phosphorylation of the eukaryotic translation initiation factor eIF4E contributes to its transformation and mRNA transport activities. Cancer Res 64: 8639-8642, 2004.

29. Rousseau D, Kaspar R, Rosenwald I, Gehrke L and Sonenberg N: Translation initiation of ornithine decarboxylase and nucleocytoplasmic transport of cyclin D1 mRNA are increased in cells overexpressing eukaryotic initiation factor 4E. Proc Natl Acad Sci USA 93: 1065-1070, 1996.

30. Ruggero D, Montanaro L, Ma L, et al: The translation factor eIF-4E promotes tumor formation and cooperates with c-Myc in lymphomagenesis. Nat Med 10: 484-486, 2004.

31. Li BD, Gruner JS, Abreo F, et al: Prospective study of eukaryotic initiation factor 4E protein elevation and breast cancer outcome. Ann Surg 235: 732-739, 2002.

32. Proud CG: Signalling to translation: how signal transduction pathways control the protein synthetic machinery. Biochem J 403: 217-234, 2007.

33. Buxade M, Parra JL, Rousseau S, et al: The Mnks are novel components in the control of TNF alpha biosynthesis and phosphorylate and regulate hnRNP A1. Immunity 23: 177-189, 2005.

34. Buxade M, Parra-Palau JL and Proud CG: The Mnks: MAP kinase-interacting kinases (MAP kinase signal-integrating kinases). Front Biosci 13: 5359-5373, 2008.

35. Niessen CM and Gottardi CJ: Molecular components of the adherens junction. Biochim Biophys Acta 1778: 562-571, 2008. 